\title{
Immunological Approach of Personalized Treatment for Recurrent Implantation Failure Patients Undergoing IVF
}

\author{
Indira Hinduja ${ }^{1,2 *}$, Amruta DS Pathare ${ }^{1}$ and Kusum Zaveri ${ }^{1}$ \\ ${ }^{1}$ Honorary Consultant Gynecologist and Infertility Specialist, P.D. Hinduja Hospital and Medical Research Centre, India \\ ${ }^{2}$ Honorary Consultant Gynecologist and Infertility Specialist, Jaslok Hospital and Research Centre, India
}

Submission: June 12, 2018; Published: July 23, 2018

*Corresponding author: Indira Hinduja, Honorary Consultant Gynecologist and Infertility Specialist, PD Hinduja Hospital and Medical Research Centre, Mumbai, India, Tel: 02267668181; Email: indirahinduja@gmail.com

\begin{abstract}
Genes related to immune and inflammatory response initiate the process of endometrial receptivity. It stimulates the cascade of biological functions like cell differentiation, vasculogenesis, and expression of cell adhesion markers at the endometrium to which the invading embryo attaches. Dysregulation in immunological response results in impairment in endometrial receptivity leading to implantation failure. In our study, we have evaluated the expression of genes involved in immune and inflammatory response in recurrent implantation failure patients and designed the personalized immunological treatment and cycle in which the embryo transfer to be recommended, based on the gene expression results. We achieved success in two out of four cases having normal expression and down-regulation of immune response respectively, in natural cycle. However, the approach is required to be implemented in larger cohort of implantation failure patients to confirm its efficiency.
\end{abstract}

Keywords: Endometrial receptivity; Implantation failure; Immune and inflammatory response; Genes; Cell differentiation; Vasculogenesis; Endometrium; Immunological treatment; Embryo transfer; Natural cycle; Window of implantation; Blastocyst; Monocytes; Natural killer cells; Tumor necrosis factor; Pro-inflammatory cytokines; Interleukin; Leukemia; Chemotaxis; Unexplained infertility; Oocytes; Immunological genes

Abbreviations: WOI: Window Of Implantation; TNF $\alpha$ : Tumor Necrosis Factor; IGF: Insulin-Like Growth Factor; PAEP: Progestogen Associated Endometrial Protein

\section{Introduction}

Immunological and inflammatory response plays very crucial role in making the endometrium receptive. In normal fertile women, under the influence of progesterone and estrogen, during the window of implantation (WOI) and before the arrival of blastocyst, immune cells like monocytes and natural killer cells migrate to the endometrium. Additionally, inflammatory factors like tumor necrosis factor (TNF $\alpha$ ) and interferon- $\gamma$ are secreted by the endometrial stromal cells to initiate the inflammatory action. These markers secrete pro-inflammatory cytokines such as interleukin (IL)1, IL6, leukemia inhibitory factor (LIF), leptin, Insulin-like growth factor (IGF)2, IL18 in the endometrial tissue [1]. The chemotaxis of monocytes to the secreted cytokines brings about the differentiation of monocytes into dendritic cells and macrophages in the presence of IL4 and Granulocytemacrophage colony-stimulating factor GMCSF. Immune cells also get differentiated to specific decidualized cells having peculiar characteristics e.g. NK cells loses its cytotoxic property when gets differentiated to decidualized NK cells. The chemotaxis and migration of these immune cells to the decidualized endometrium stimulates the endometrial cells to secrete various chemokines, growth factors and expression of cell adhesion molecules. Thus, the immunological and adequate inflammatory response has been observed to plays vital role in decidualization, tissue remodeling, angiogenesis, vasculogenesis, and cell adhesion.

Based on the study performed by our group [2], it has been observed that the immunological and inflammatory genes were downregulated in recurrent implantation failure patients undergoing 2 or more IVF cycles with unexplained infertility. The down-regulated expression of these genes was observed in endometrial tissue when patient group as well as healthy fertile oocyte donors (control group) were under the influence of ovarian stimulation. We have shortlisted panel of 70 genes involved in immune and inflammatory response. However, genes such as Progestogen Associated Endometrial Protein (PAEP), CD4, C-X-C Motif Chemokine Ligand 14 (CXCL14), Interleukin 6 Signal Transducer (IL6ST), LIF, Phospholipase A2 Group IIA (PLA2G2A), 


\section{Global Journal of Reproductive Medicine}

were validated by real time PCR. The protein expression was also assessed by immunohistochemistry for LIF, IL6ST and PAEP gene, confirming the significant down-regulation [2].

The significant differential expression of these genes in RIF cases Vs oocyte donors highlights the role of immunological processes in endometrial receptivity. Therefore, impairment in immune response and imbalance between pro-inflammatory and anti-inflammatory response could be accountable for defective endometrial receptivity [1-3]. It has been reported that, the hormonal administration in IVF protocol can be detrimental to the endometrial receptivity [4]. Though in our previous study, this hormonal bias was ruled out by considering both the case and control group undergoing same protocol of ovarian stimulation, the protocol of embryo transfer would also affect the receptivity. Thus, the regulation of immune and inflammatory cells in stimulated cycle, hormonal replacement cycle and natural cycle would be different which may cause implantation failure.

Therefore, in each RIF patients, it is essential to evaluate the expression of immune and inflammatory markers in WOI of the same characterized cycle as of embryo transfer. Based on the evaluation of panel of immunological markers, the approach of personalized treatment can be planned for RIF patients. In this approach, the RIF patients had undergone the evaluation of panel of markers involved in immunological response such as PAEP, LIF, IL6ST, CXCL14, PLA2G2A, Indoleamine 2,3-Dioxygenase 1 (ID01), CD4, CD247. On stimulating the ovaries and retrieval of the oocytes, the embryos of RIF patients were fertilized and cryopreserved until their transfer. In the next natural menstrual cycle, the endometrial biopsy was collected on day LH+6, confirming the ovulation by monitoring the follicular growth by USG and LH surge by 'ovulation LH kit'. Total RNA was extracted from endometrial tissue using Trizol reagent and were subjected to cDNA preparation using 'first strand cDNA kit", Invitrogen as per the manufacturer's instructions. Real time PCR was performed using $18 \mathrm{~S}$ housekeeping gene as a normalizer. The CT values of these genes were compared to control groups i.e. healthy fertile women in natural cycle $(\mathrm{LH}+6$ th day). The fold change was calculated using $2^{\Delta \Delta \mathrm{CT}}$ method. The down-regulation or upregulation of these markers having fold change $>2$ was considered abnormal and were subjected to further personalized treatment.

The down regulation indicates the need to elicit the inflammatory response in endometrium by scratching the endometrium or introducing injury to endometrium using Gynetics suction curette. In such cases we could use the mechanistic approach reported by Gnainsky et al. [3], in which induced endometrial injury stimulates the immune markers and their migration to the implantation site, which reside in the functionalis layer of the endometrium and gets activated in next menstrual cycle during WOI under the influence of steroid hormones. On the other hand, the up-regulation of above mentioned immune markers is suggestive over-expression of genes related to immune response and would be recommended the administration of immunosuppressant (Figure 1). In all the down-regulated, up-regulated and normal cases, the embryo transfer is to be performed in natural cycle on day $\mathrm{LH}+5$ by monitoring the follicular growth, without giving any hormonal supplementation for preparation of endometrium.

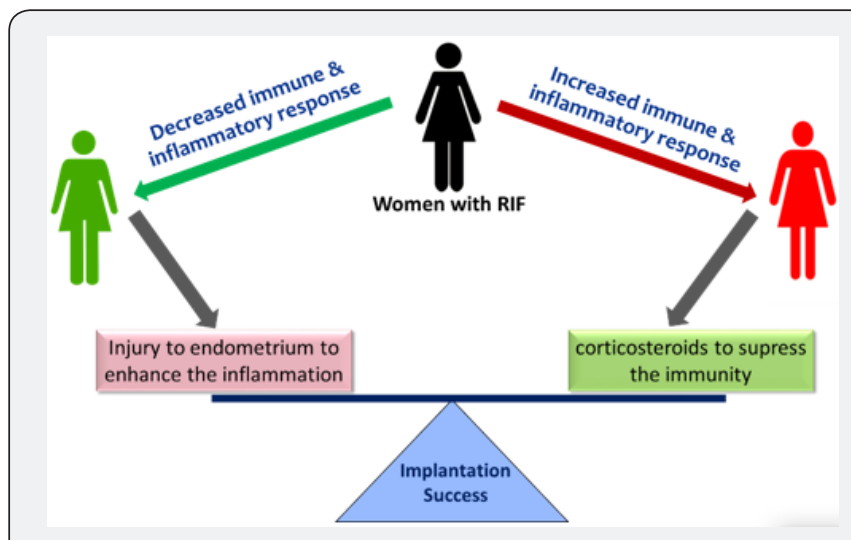

Figure 1: Personalized treatment approach for recurrent implantation failure patients.

This approach agrees with the study reported by Roberson et al. [5] suggesting that it is very essential to treat the infertility immunologically by giving corticosteroids, only after the evaluation of immune response in the endometrium. This is because the controlled inflammation and stimulation of immune cells are important factors to achieve receptivity and successful implantation [5]. We have evaluated four such cases, of which one showed down-regulated panel of immune markers who had undergone the 'injury to endometrium' in secretory phase. In this patient, the embryos were transferred in its next natural cycle. In other case, the panel of immunological genes were normal when compared to fertile women. In this case, the embryos were transferred in the next natural cycle. Both these patients had conceived having positive urine pregnancy test and the presence of gestational sack. The conception was confirmed by assessment of heart bits of the fetus. Hence, out of four, IVF was successful in two patients on managing them using personalized approach with respect to immunological status of the endometrium to achieve the receptivity. The fold change of both these patients is shown in Table 1.

Table 1: Fold change of two patients achieved paregnancy after implementation of approach of personalized treatment. Patient 1 showed normal expression having fold change of maximum markers < 2 whereas patient 2 observed to have down-regulation of immune and inflammatory response having downregulated fold change of $>2$.

\begin{tabular}{|c|c|c|}
\hline Genes & Fold change Patient 1 & Fold change Patient 2 \\
\hline PAEP & 0.295 & -7.857 \\
\hline PLA2G2A & 2.105 & -4.26 \\
\hline CD4 & 0.09 & -0.414 \\
\hline CXCL14 & 0.7 & -8.254 \\
\hline IDOL1 & 1.145 & 2.594 \\
\hline CD247 & 1.57 & 0.58 \\
\hline IL6ST & 1.57 & -2.957 \\
\hline LIF & -1.36 & -3.89 \\
\hline
\end{tabular}




\section{Conclusion}

The implementation of this approach needs to be substantiated in larger population of RIF patients. Thus, the immune response and its proper regulation is observed to play vital role in endometrial receptivity. In IVF, the stated approach of embryo transfers in natural cycle on assessment of immunological regulatory genes would help in improving the success rate of IVF. The endometrial tissue assessment in natural cycle and even the transfer of embryo in natural cycle would help to nullify the hormonal bias of other generalized protocols of ovarian stimulation, replacement therapy and the preparation of endometrium in frozen embryo transfer cycle.

\section{Aknowledgement}

We are grateful to P.D. Hinduja Hospital and Medical Research centre as well as National Health and education Society for santioning of the project.

\section{References}

1. Vinketova K, Mourdjeva M, Oreshkova T (2016) Human Decidual Stromal Cells as a Component of the Implantation Niche and a Modulator of Maternal Immunity. J Pregnancy doi: 10.1155/2016/8689436.

2. Pathare ADS, Zaveri K, Hinduja I (2017) Downregulation of genes related to immune and inflammatory response in IVF implantation failure cases under controlled ovarian stimulation. Am J Reprod Immunol 78(1): e12679.

3. Gnainsky Y, Granot I, Aldo P, Barash A, Or Y, et al. (2015) Biopsyinduced inflammatory conditions improve endometrial receptivity: The mechanism of action. Reproduction. 149(1): 75-85.

4. Horcajadas JA, Riesewijk A, Polman J, van Os R, Pellicer A, et al. (2005) Effect of controlled ovarian hyperstimulation in IVF on endometrial gene expression profiles. Mol Hum Reprod 11(3): 195-205.

5. Robertson SA, Jin M, Yu D, Moldenhauer LM, Davies MJ, et al. (2016) Corticosteroid therapy in assisted reproduction-immune suppression is a faulty premise. Hum Reprod 31(10): 1-10.

Your next submission with Juniper Publishers
will reach you the below assets
- Quality Editorial service
- Swift Peer Review
- Reprints availability
- E-prints Service
- Manuscript Podcast for convenient understanding
- Global attainment for your research
- Manuscript accessibility in different formats
( Pdf, E-pub, Full Text, Audio)
- Unceasing customer service
Track the below URL for one-step submission
https://juniperpublishers.com/online-submission.php

